\title{
Versatile voltage-mode multifunction biquadratic filter employing DDCCs
}

\author{
H. P. Chen ${ }^{\text {a) }}$ and P. L. Chu \\ Department of Electronic Engineering, De Lin Institute of Technology, No. 1, \\ Lane 380, Qingyun Rd., Tucheng City, Taipei \\ a)hpchen@dlit.edu.tw
}

\begin{abstract}
A new versatile voltage-mode biquadratic with four inputs and four outputs using two differential difference current conveyors (DDCCs), two grounded capacitors, and two resistors is proposed. The proposed circuit can act as a multifunction voltage-mode filter with one or three inputs and four outputs and can simultaneous realization of voltage-mode notch, highpass, bandpass and lowpass filter signals from the four output terminals, respectively, without any component choice conditions. On the other hand, it also can act as a universal voltagemode filter with three inputs and a single output and can realize five generic voltage-mode filter signals from the same configuration without any component matching conditions. H-Spice and MATLAB simulations results are provided to demonstrate the theoretical analysis.
\end{abstract}

Keywords: active filters, current conveyors, analog electronics

Classification: Integrated circuits

\section{References}

[1] W. J. Horng, J. R. Lay, C. W. Chang, and M. H. Lee, "High input impedance voltage-mode multifunction filters using plus-type CCII's," Electron. Lett., vol. 33, pp. 472-473, 1997.

[2] C. M. Chang, "Multifunction biquadratic filters using current conveyors," IEEE Trans. Circuits Syst. II, vol. 44, pp. 956-958, 1997.

[3] C. M. Chang and M. J. Lee, "Voltage-mode multifunction filter with single input and three outputs using two compound current conveyors," IEEE Trans. Circuits Syst. I, vol. 46, pp. 1364-1365, 1999.

[4] C. M. Chang, B. M. Al-Hashimi, C. L. Wang, and C. W. Hung, "Single fully differential current conveyor biquad filters," IEE Proc. Circuits, Devices and Syst., vol. 150, pp. 394-398, 2003.

[5] J. W. Horng, W. Y. Chui, and H. Y. Wei, "Voltage-mode highpass, bandpass and lowpass filters using two DDCCs," Int. J. Electron., vol. 91, pp. 461-464, 2004.

[6] J. W. Horng, C. C. Tsai, and M. H. Lee, "Novel universal voltage-mode biquad filter with three inputs and one output using only two current conveyors," Int. J. Electron., vol. 80, pp. 543-546, 1996.

[7] J. W. Horng, M. H. Lee, H. C. Cheng, and C. W. Chang, "New CCII-based voltage-mode universal biquadratic filter," Int. J. Electron., vol. 82, pp. 151-155, 1997.

[8] S. I. Liu and J. L. Lee, "Voltage-mode universal filters using two current 
conveyors," Int. J. Electron., vol. 82, pp. 145-149, 1997.

[9] J. W. Horng, "Voltage-mode multifunction filter using one current feedback amplifier and one voltage follower," Int. J. Electron., vol. 88, pp. 153-157, 2001.

[10] J. W. Horng, "High-input impedance voltage-mode universal biquadratic filter using three plus-type CCIIs," IEEE Trans. Circuit Syst. II, vol. 48, pp. 996-997, 2001.

[11] J. W. Horng, "Voltage-mode universal biquadratic filters using CCIIs," IEICE Trans. Fundamentals, vol. E87-A, pp. 406-409, 2004.

[12] C. M. Chang and H. P. Chen, "Universal capacitor-grounded voltagemode filter with three inputs and a single output," Int. J. Electron., vol. 90, pp. 401-406, 2003.

\section{Introduction}

The applications and advantages in the realization of various active filter transfer functions using current conveyors have received considerable attention. Some voltage-mode multifunction second-order filters with a single input and three outputs using current conveyors were proposed $[1,2,3,4,5]$. In 1997, Horng et al. [1] proposed a voltage-mode multifunction filter with a single input and three outputs, which can realize lowpass, bandpass, and highpass filter transfer functions, using four $\mathrm{CCII}+\mathrm{s}$, three grounded capacitors, and five grounded resistors. In the same year, Chang proposed five voltage-mode multifunction biquadratic filters using four CCIIs, two grounded capacitors, and three-five resistors [2]. However, with these two proposed configurations required at least four active components. In 1999, Chang and Lee proposed a voltage-mode lowpass, bandpass, and highpass biquadratic filter with a single input and three outputs using only two compound current conveyors, two grounded capacitors, and three resistors [3]. However, the passive components it used were not minimum. In 2003, Chang et al. [4] proposed a voltage-mode multifunction filter with a single input and four outputs, which can realize lowpass, bandpass, and highpass filter transfer functions, using a single FDCCII, two grounded capacitors, and two grounded resistors. However, only three standard filter signals can be simultaneously obtained in the circuit configuration. Moreover, the use of FDCCII can be divided into two separate DDCC's by using $Y_{1}, Y_{2}, Y_{3}, \mathrm{X}+$ and $\mathrm{Z}+$ as the terminals of the first DDCC, and $Y_{1}, Y_{2}, Y_{4}, \mathrm{X}$ - and Z- as the terminals of the second DDCC. Recently, Horng et al. [5] proposed a voltage-mode highpass, bandpass and lowpass filters using two DDCCs, two grounded capacitors, and two grounded resistors. However, only three standard filter signals can be simultaneously obtained in the circuit configuration. In this paper, the authors also employ the same active elements of DDCC, but the proposed circuit can realize more "notch" filter signal from the same configuration, which is unlike the proposed by Horng et al. [5]. On the other hand, many universal voltage-mode biquads with three inputs and one output were proposed $[6,7,8,9,10,11]$. However, with these proposed circuits could 
not used grounded capacitors in the circuit design. In 2003, Chang and Chen [12] proposed a good universal voltage-mode filter with three inputs and one output employing two DDCCs, two grounded capacitors and two resistors. However, only three inputs and single output was used in circuit design. In this paper, the authors propose a voltage-mode biquad with four inputs and four outputs using only two DDCCs, two grounded capacitors, and two resistors. The proposed circuit can act as a multifunction voltagemode filter with one or three inputs and four outputs and can simultaneous realization voltage-mode notch, highpass, bandpass and lowpass filter signals from the four output terminals, respectively. On the other hand, it can also act as a universal voltage-mode filter with three inputs and a single output and can realize five generic voltage-mode filter signals from the same configuration without any component matching conditions. Therefore, the proposed circuit is obviously more versatile than those with a single input and multi-output or with multi-input and a single output.

\section{Circuit description}

The proposed versatile voltage-mode multifunction biquadratic filter with four inputs and four outputs employing two DDCCs is shown in Fig. 1. By using standard notation, the port relations of a DDCC can be characterized by $I_{Y 1}=I_{Y 2}=I_{Y 3}=0, V_{X}=V_{Y 1}-V_{Y 2}+V_{Y 3}$, and $I_{Z}= \pm I_{X}[5,12]$. The proposed circuit comprises two DDCCs, two grounded capacitors and two resistors, to realize the voltage-mode multifunction biquadratic filter. The use of grounded capacitors is particularly attractive for integrated circuit implementation. Derived by each nodal equation of the proposed circuit that the input-output relationship matrix form of Fig. 1 can be expressed as

$$
\left[\begin{array}{cccc}
s C_{1} & 0 & G_{1} & 0 \\
0 & s C_{2} & 0 & G_{2} \\
-1 & -1 & 1 & 0 \\
1 & 0 & 0 & 1
\end{array}\right]\left[\begin{array}{c}
V_{o 1} \\
V_{o 2} \\
V_{o 3} \\
V_{o 4}
\end{array}\right]=\left[\begin{array}{c}
G_{1} V_{i 1} \\
G_{2} V_{i 3} \\
-V_{i 2} \\
V_{i 1}+V_{i 4}
\end{array}\right]
$$

where $G_{1}=\frac{1}{R_{1}}$ and $G_{2}=\frac{1}{R_{2}}$.

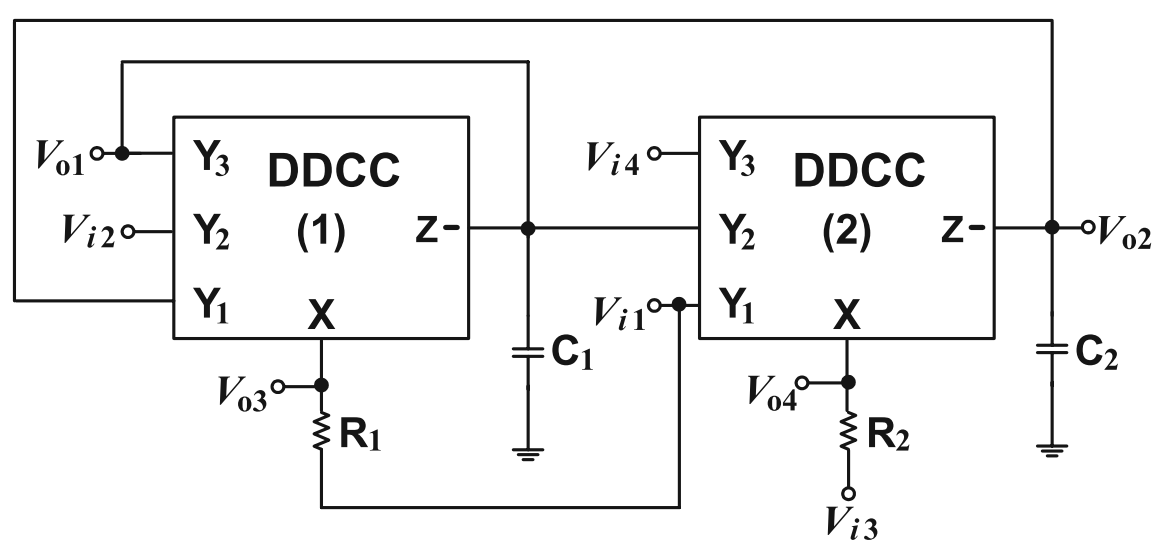

Fig. 1. The proposed multifunction voltage-mode biquad. 
From the above matrix form, the following four output voltages can be derived as

$$
\begin{gathered}
V_{o 1}=\frac{1}{\triangle}\left[\left(s C_{2} G_{1}+G_{1} G_{2}\right) V_{i 1}+s C_{2} G_{1} V_{i 2}-G_{1} G_{2} V_{i 3}+G_{1} G_{2} V_{i 4}\right] \\
V_{o 2}=\frac{1}{\triangle}\left[s C_{1} G_{2} V_{i 1}+G_{1} G_{2} V_{i 2}+\left(s C_{1} G_{2}+G_{1} G_{2}\right) V_{i 3}-\left(s C_{1} G_{2}+G_{1} G_{2}\right) V_{i 4}\right] . \\
V_{o 3}=\frac{1}{\triangle}\left[\left(s C_{2} G_{1}+s C_{1} G_{2}+G_{1} G_{2}\right) V_{i 1}-s^{2} C_{1} C_{2} V_{i 2}+s C_{1} G_{2} V_{i 3}-s C_{1} G_{2} V_{i 4}\right] \\
V_{o 4}=\frac{1}{\triangle}\left[s^{2} C_{1} C_{2} V_{i 1}-s C_{2} G_{1} V_{i 2}+G_{1} G_{2} V_{i 3}+\left(s^{2} C_{1} C_{2}+s C_{2} G_{1}\right) V_{i 4}\right] . \\
\text { where } \triangle=s^{2} C_{1} C_{2}+s C_{2} G_{1}+G_{1} G_{2} .
\end{gathered}
$$

Depending on the status of the biquad input four voltages: $V_{i 1}, V_{i 2}, V_{i 3}$, and $V_{i 4}$, numerous filter functions are obtained. There are three cases shown as below.

Case I. If $V_{i 2}=V_{i n}$ (the input voltage signal) and $V_{i 1}=V_{i 3}=V_{i 4}=0$ (namely, the resistors $R_{1}$ and $R_{2}$ are grounded), then

$$
\begin{aligned}
\frac{V_{o 1}}{V_{i n}} & =\frac{s C_{2} G_{1}}{s^{2} C_{1} C_{2}+s C_{2} G_{1}+G_{1} G_{2}} . \\
\frac{V_{o 2}}{V_{i n}} & =\frac{G_{1} G_{2}}{s^{2} C_{1} C_{2}+s C_{2} G_{1}+G_{1} G_{2}} . \\
\frac{V_{o 3}}{V_{i n}} & =\frac{-s^{2} C_{1} C_{2}}{s^{2} C_{1} C_{2}+s C_{2} G_{1}+G_{1} G_{2}} . \\
\frac{V_{o 4}}{V_{i n}} & =\frac{-s C_{2} G_{1}}{s^{2} C_{1} C_{2}+s C_{2} G_{1}+G_{1} G_{2}} .
\end{aligned}
$$

Thus, the non-inverting bandpass, non-inverting lowpass, inverting highpass, and inverting bandpass signals are obtained at the node voltages, $V_{o 1}$, $V_{o 2}, V_{o 3}$, and $V_{o 4}$, respectively. Note that the input signal, $V_{i 2}=V_{i n}$, is connected to the high input impedance input node of the DDCC(1) (the $Y_{2}$ port of the DDCC(1)). So the circuit enjoys the advanage of having high input impedance, leading to cascadability at the input port. Moreover, the use of only grounded capacitors and resistors is particularly attractive for integrated circuit implementation.

Case II. If $V_{i 2}=V_{i 3}=V_{i 4}=V_{i n}$ (the input voltage signal) and $V_{i 1}=0$, then

$$
\begin{aligned}
& \frac{V_{o 1}}{V_{i n}}=\frac{s C_{2} G_{1}}{s^{2} C_{1} C_{2}+s C_{2} G_{1}+G_{1} G_{2}} . \\
& \frac{V_{o 2}}{V_{i n}}=\frac{G_{1} G_{2}}{s^{2} C_{1} C_{2}+s C_{2} G_{1}+G_{1} G_{2}} . \\
& \frac{V_{o 3}}{V_{i n}}=\frac{-s^{2} C_{1} C_{2}}{s^{2} C_{1} C_{2}+s C_{2} G_{1}+G_{1} G_{2}} . \\
& \frac{V_{o 4}}{V_{i n}}=\frac{s^{2} C_{1} C_{2}+G_{1} G_{2}}{s^{2} C_{1} C_{2}+s C_{2} G_{1}+G_{1} G_{2}} .
\end{aligned}
$$

Thus, the non-inverting bandpass, non-inverting lowpass, inverting highpass, and non-inverting notch signals are obtained at the node voltages, 
$V_{o 1}, V_{o 2}, V_{o 3}$, and $V_{o 4}$, respectively. Note that one more "notch" signal by Horng. [5]

Case III. Let $V_{i 4}=0$ from Eq. (5) yield the five types of biqudratic filters with the following specialization.

(i) lowpass: $V_{i 1}=V_{i 2}=0$, and $V_{i 3}=V_{i n}$;

(ii) bandpass: $V_{i 1}=V_{i 3}=0$, and $V_{i 2}=V_{i n}$;

(iii) highpass: $V_{i 2}=V_{i 3}=0$, and $V_{i 1}=V_{i n}$;

(iv) notch: $V_{i 2}=0$, and $V_{i 1}=V_{i 3}=V_{i n}$;

(v) allpass: $V_{i 1}=V_{i 2}=V_{i 3}=V_{i n}$.

Note that there is also no need of any component matching conditions and inverting type voltage input signal to realize all of the filter responses. Obviously, from case I to III, the proposed circuit can act as a multifunction voltage-mode filter with one or three inputs and four outputs and it can also act as a universal voltage-mode filter with three inputs and a single output, too. Therefore, the proposed circuit is more versatile than those with a single input and three outputs or with multi-input and a single output.

\section{Effect of Non-idealities}

Taking into account the non-idealities of a DDCC, namely the $V_{X}=\beta_{a} V_{Y 1}-$ $\beta_{b} V_{Y 2}+\beta_{c} V_{Y 3}$, and $I_{Z}=-\alpha I_{X}$ where $\alpha_{i}=1-e_{i}$ and $e_{i}\left(\left|e_{i}\right| \ll 1\right)$ denote the current tracking error and $\beta_{v}=1-e_{v}$ and $e_{v}\left(\left|e_{v}\right| \ll 1\right)$ denote the differential voltage tracking error. The denominator of the transfer functions of Fig. 1 becomes

$$
D(s)=s^{2} C_{1} C_{2}+\alpha_{1} \beta_{c 1} s C_{2} G_{1}+\alpha_{1} \alpha_{2} \beta_{a 1} \beta_{b 2} G_{1} G_{2} .
$$

The non-ideal $\omega_{o}$ and $Q$ are given by

$$
\begin{aligned}
& \omega_{o}=\sqrt{\frac{\alpha_{1} \alpha_{2} \beta_{a 1} \beta_{b 2} G_{1} G_{2}}{C_{1} C_{2}} .} \\
& Q=\frac{1}{\beta_{c 1}} \sqrt{\frac{\alpha_{2} \beta_{a 1} \beta_{b 2} C_{1} G_{2}}{\alpha_{1} C_{2} G_{1}}} .
\end{aligned}
$$

A sensitivity study forms an important index of the performance of any active network. The formal definition of sensitivity is

$$
S_{x}^{F}=\frac{x}{F} \frac{\partial F}{\partial x} .
$$

where $F$ represents one of $\omega_{o}, Q$ and $x$ represents any of the passive elements $\left(G_{1}-G_{2}, C_{1}-C_{2}\right)$ or the active parameters $\left(\alpha_{i}, \beta_{i}\right)$. Using the above definition the active and passive sensitivities of the proposed circuit shown in Fig. 1 are given as

$$
\begin{gathered}
S_{\alpha_{1}}^{\omega_{o}}=S_{\alpha_{2}}^{\omega_{o}}=S_{\beta_{a 1}}^{\omega_{o}}=S_{\beta_{b 2}}^{\omega_{o}}=S_{G_{1}}^{\omega_{o}}=S_{G_{2}}^{\omega_{o}}=-S_{C_{1}}^{\omega_{o}}=-S_{C_{2}}^{\omega_{o}}=\frac{1}{2} \\
S_{\alpha_{2}}^{Q}=S_{\beta_{a 1}}^{Q}=S_{\beta_{b 2}}^{Q}=S_{C_{1}}^{Q}=S_{G_{2}}^{Q}=-S_{\alpha_{1}}^{Q}=-S_{C_{2}}^{Q}=-S_{G_{1}}^{Q}=\frac{1}{2} \\
S_{\beta_{c 1}}^{Q}=-1 .
\end{gathered}
$$

all of which are low and not larger than unity in absolute value. 


\section{Simulation results}

In order to verify the theoretical prediction of the proposed biquad filter, we use the H-Spice for the simulation part with TSMC $0.35 \mu \mathrm{m}$ process and Mat-lab for the theoretical part to compare the results. The CMOS implementation of a DDCC as shown in [12] with the NMOS and PMOS transistor aspect ratios $(W / L=5 \mu / 1 \mu)$ and $(W / L=10 \mu / 1 \mu)$, respectively. The supply voltages were $V_{D D}=-V_{S S}=1.65 \mathrm{~V}$, and the biasing voltages were $V_{b}=-V_{b 1}=0.5 \mathrm{~V}$. Figure 2 shows the result of circuit simulation for the proposed multifunction filter in case I. The component values are selected as $R_{1}=10 \mathrm{k} \Omega, R_{2}=2 \mathrm{k} \Omega, C_{1}=79.5 \mathrm{pF}$, and $C_{2}=15.9 \mathrm{pF}$ leads to a center frequency of $f_{o}=1 \mathrm{MHz}$ and quality factor of $Q=5$. As can be seen, there is a close agreement between theory and simulation.

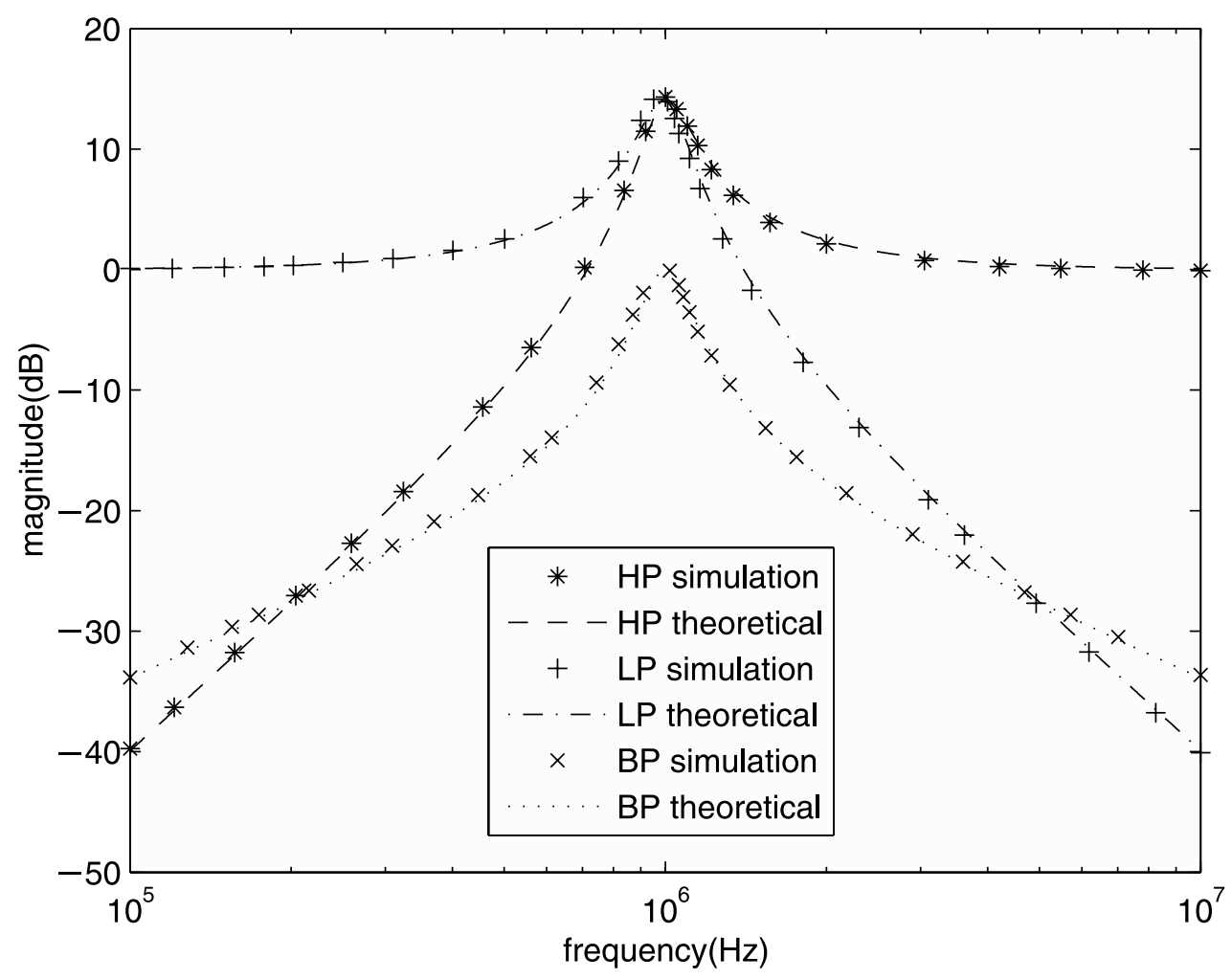

Fig. 2. The simulated results of lowpass, bandpass, and highpass amplitude-frequency responses with $V_{i 2}=V_{i n}$, and $V_{i 1}=V_{i 3}=V_{i 4}=0$.

\section{Conclusion}

In this paper, we presented a new versatile multifunction voltage-mode biquadratic filter with four inputs and four outputs. The circuit can be acted as both a multifunction voltage-mode filter with one or three inputs and four outputs and a universal voltage-mode filter with three inputs and a single output. Therefore, the circuit proposed in this paper is more versatile than the multifunction one with a single input and three outputs or the 
universal one with three inputs and a single output. Besides, the proposed circuit still offers the following advantages: (i) simultaneous realization of lowpass, bandpass, and highpass responses for the single-input three-output filter, (ii) simultaneous realization of lowpass, bandpass, highpass and notch responses from the four output terminals, respectively, (iii) no need to impose component choice to realize all five generic responses for the three-input single-output filter, (iv) no need to employ inverting-type input signals, (v) employment only two grounded capacitors, and (vi) low active and passive sensitivity performances. In Table I, the main features of the proposed new circuit are compared with those of previous works.

Table I. Performance parameters of recently reported voltage-mode filters.

\begin{tabular}{|c|c|c|c|c|c|c|}
\hline \multirow{2}{*}{ Circuits } & \multicolumn{5}{|c|}{ Criteria } \\
\cline { 2 - 6 } & (i) & (ii) & (iii) & (iv) & (v) & (vi) \\
\hline The new circuit & yes & yes & yes & yes & yes & yes \\
Ref. [12] in 2003 & no & no & yes & yes & yes & yes \\
Ref. [11] in 2004 & no & no & yes & no & no & no \\
Ref. [10] in 2001 & no & no & yes & no & no & no \\
Ref. [9] in 2001 & no & no & yes & no & no & yes \\
Ref. [5] in 2004 & yes & no & no & yes & yes & yes \\
Ref. [4] in 2003 & yes & no & no & yes & yes & yes \\
Ref. [3] in 1999 & yes & no & no & yes & yes & yes \\
\hline
\end{tabular}

\section{Acknowledgments}

The authors would like to thank the National Science Council and Chip Implementation Center of Taiwan, ROC. 